No. I. Katze von $1870 \mathrm{~g}$. Vormittags $8^{\text {h }} 55^{\prime} \quad 0,3 \mathrm{~g}$ Chloreton durch Schlundsonde. Adrenalinlösung $1: 10 \mathrm{NaCl}$-lösung. $50 \mathrm{~cm}$ Ueberdruck.

\section{Intravenöse Adrenalin-Dauerinfusion.}

\section{Von Ed. Koll in Barmen.}

Der Fall einer 28 jährigen Patientin meiner Abteilung, die wegen unerträglicher tabischer Magenkrisen der Försterschen Operation unterzogen wurde und unter den Zeichen der Splanchnikuslähmung starb, gab mir Veranlassung, dem Versuche einer intravenösen Adrenalin-Dauerinfusion bei schwerem Herz- und Gefäßkollapse näherzutreten. Die diskontinuierliche Anwendung führt bekanntlich nur zu einer überaus flüchtigen Blutdrucksteigerung, da das Adrenalin unter dem Einfluß des Blutalkalis sehr schnell unwirksam wird. An die Frage, ob eine anhaltende Wirkung bei ununterbrochener Zufuhr zu erwarten ist, oder ob der Tierkörper ïber kurz oder lang refraktär gegen die weitere Zufuhr wird, ist Kretseh mer in seinen bemerkenswerten Tierversuchen herangetreten; es zeigte sich, daß ein refraktäres Stadium nicht eintritt, daß vielmehr bei Dauerzufuhr eine gleichmäßige Dauerwirkung auf die Blutdruckkurve resultiert, die während der bis zu drei Stunden ausgedehnten Versuche stets proportional der Einflußgeschwindigkeit, d. h. der eingeflossenen Adrenalinmenge war.

Eine Bestätigung dieser Ergebnisse schien mir vor der Uebertragung der Dauerinfusionen ans Krankenbett erforderlich. Diesem Zwecke dient eine Reihe von Versuchen an Katzen, die ich im Pharmakologischen Institute der Farbwerke vormals Fr. Bayer \& Co. in Elberfeld ausführte, dessen Leiter, Herr Prof. Dres er, mir in freundlichster Weise seinen Rat und seine Hilfsmittel zur Verfügung stellte. Tch gebe über die Versuche nur einen kurzen Bericht.

Ich benutzte das Suprareninum syntheticum der Firma Meister, Lucius \& Brüning, das diese mir in liberaler Weise $\mathrm{zu}$ meinen Versuchen überließ. Zur Narkose und experimentellen Blutdruckherabsetzung verwandte ich das Chloreton, das nach der Untersuchung von Impens in erster Linie durch Lähmung des Vasomotorenzentrums den Blutdruck herabsetzt, während die herzmuskelschädigende Wirkung in zweiter Linie steht. Im Versuch war die linke Carotis ext. mit Kymographion verbunden und in die gleichseitige Vena jugularis die Infusionskanüle eingefügt. Der zweckmäßigste Grad der Verdünnung und die zu einer kräftigen Wirkung nach Eintritt der toxischen Blutdrucksenkung erforderliche Dosis wurde im Vorversuch durch diskontinuierliche Injektion mit Rekordspritze ermittelt. Es ergab sich dabei Folgendes: Die Latenzzeit ist ganz kurz, ein bis zwei Sekunden, dann steigt der Blutdruck unabhängig von der injizierten Adrenalinmenge steil binnen acht bis zehn Sekunden in die Höhe bis zu seinem Maximum; dann allmählicher Abfall. Die Rückkehr zur Norm vollzieht sich schneller oder langsamer proportional der auf einmal injizierten Adrenalinmenge:

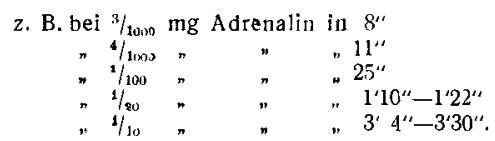

Diese Beobachtungen entsprechen den bereits von Kretschmer gemachten Feststellungen.

Die Dauerinfusionen geschehen nach folgender Anordnung

An einem mit Zentimeterskala versehenen Stativ ist der neben. stehend abgebildete kleine Apparat mit gleichbleibendem Flüssigkeitsniveau, dessen Zusammensetzung ohne weiteres ersichtlich ist, in der Höhe leicht von $0-100 \mathrm{~cm}$ über Niveau der zur Infusion benützten Vene verschieblich, angebracht. Zur Füllung wird Schlauch a in die Adrenalinkochsalzmischung getaucht und an Schlauch $b$ nach Lockerung der Klemme mit dem Munde langsam aspiriert. Schlauch e führt zur Vene. Als Venenkanüle benutzte ich eine dünne, sorgfältig abgestumpfte und geglättete Hohlnadel (am besten Platin-Iridiumnadel); oberhalb der Kanüle ist, wie in den $\mathrm{K}_{\mathrm{r}}$ e ts $\mathrm{ch}$ mer schenVersuchen, ein Glasröhrchen mit kapillarer Strecke (von der Firma Haack in Jena) eingefiigt, die in einer Minute bei $50 \mathrm{~cm}$ Ueberdruck ctwa $1 \mathrm{~cm}$ Adrenalinkochsalzlösung durchfließen läßt. Es läßt sich durch diese Anordnung cler $\mathrm{Zu}-$ fluß des Adrenalins bequem und genügend ausgiebig variieren.

Aus den Daten meiner fünf Versuche gebe ich in folgendern clie der zwei längstdauernden in kurzen Auszügen wieder:

Der Blutdruck der Katze betrïgt in der Norm etwn $120 \mathrm{mmn} \mathrm{Hg}$.

No. V. Katze von $1930 \mathrm{~g}$. $8^{\text {h }} 40^{\prime} 0,3$ g Chloreton per Schlund sonde.

Adrenalinlösung I: $10 \mathrm{NaCl}-$ lösung. $50 \mathrm{~cm}$ Ueberdruck.

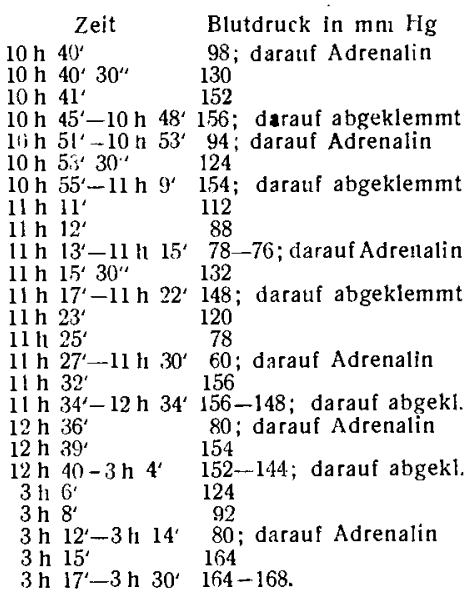

$3^{\text {h }} 50^{\prime}$ beginnt 'Tier aus Narkose zu erwachen; deshalb per Schlund sonde $0,3 \mathrm{~g}$ Chloreton; sofort darauf heftiges Erbrechen mit Aspiration: trotz sofortiger Elevation des Unterkörpers und künstlicher Atmung Exitus.

Bei der Sektion (Dr. Binder) findet sich in einem Ast der Pulmonalarterie des rechten Unterlappens ein $2 \frac{1}{1} \mathrm{~mm}$ langer Embolus; Gefäß dahinter leer.

Im ganzen sind im Laufe des 5 Stunden 10 Minuten andauernden Versuches $56,4 \mathrm{~cm}$ Adrenalinmischung, entsprechend $5,64 \mathrm{mg}$ Suprareninum syntheticum ein geflossen.

Ich übergehe die übrigen Versuche, die ganz gleichsinnig verliefen, aber infolge von Störungen der Narkose kürzer waren (20 Minuten, $11 / 4$ und $2 \frac{1}{2}$ Stunden).

In meinen sämtlichen Versuchen konnte ich dieselle durchaus gleichmäßige prompte Wirkung auf die Blutdruckkurve während der ganzen Beobachtungszeit feststellen. Eine deutliche und anhaltende Wirkung auf Frequenz und Amplitude des Pulses trat nur in zwei Fällen ein, z. B. im Versuche 5: Pulsfrequenz ohne Adrenalin 150, Amplitude $3 \mathrm{~mm}$, auf Höhe der Adrenalinwirkung 90, Amplitude $7 \mathrm{~mm}$; Pulsverlangsamung und Amplitudensteigerung gleichen sich nach Abstellung des Zuflusses langsam wieder aus. Aenderungen der Atmungsfrequenz und -tiefe wurden nicht beobachtet. Auf pharmakologische Einzelheiten gehe ich hier nicht ein.

Die Kretschmerschen Versuche im Verein mit den meinigen geben uns meines Erachtens die Berechtigung, von Adrenalindauerinfusionen in geeigneten Fällen am Krankenbett Gebrauch zu machen. Sie geben uns die Hoffnung, dadurch den peinlichen Jebelstand der Adrenalinanwendung, die überaus große Fliichtigkeit der Wirkung, auszuschalten. Ich bin deshalb seit kurzer Zeit zu therapeutischen Versuchen übergegangen und habe mich dazu derselben Anordnung bedient wie in meinen Tierversuchen. Unter Kontrolle durch Blutdruckmessung ist es dabei durch entsprechende Variierung der Einflußgeschwindigkeit möglich, die jeweilig zweckentsprechende Dosierung zu ermitteln. Ich bezweifle aber nicht, daß eine Vereinfachung der Anordnung möglich sein wird

Ich verfüge vorläufig nur über die ersten tastenden Versuche, will diese aber schon jetzt im kurzen Auszuge bekannt geben, uml eventuell weitere Velsuche anzuregen.

Fall 1. 64jährige, sehr dekrepide Frau mit großem linkseitige' Nierentumor: Puls kaum fühlbar, Druck nicht meBbar, leich te Somno Innz. Sobald die ersten Tropfen Adrenalinmischung ( 1 : $3 \mathrm{NaCl}$ - lösung $50 \mathrm{~cm}$ Ueberdruck) einfließen, Uebelkeit und Erbrechelı, Puls unfühlbar; bei Aussetzen des Adrenalinzuflusses versehwindet Uebelkeit und Er- 
brechen schnell, tritt aber sofort nach Wiederherstellung des Adrenalinzuflusses wieder auf. Versuch wird deshalb abgebrochen.

Fall 2. Mann von 61 Jahren, 27. Juli mit schwerster Kompensationsstörung des Herzens einoreliefert. Blutdruck $60 \mathrm{~mm} \mathrm{Hg}$, Puls 164 , Respiration 32, ord.

Adrenaliniufusion

$1: 3 \mathrm{NaCl}-\mathrm{L}$ jösung, 50 cem Ueberdiuek.

Von $2^{1} 2^{\prime}-2^{\prime \prime} 16^{\prime}$ fließen 5 ccns ein $2^{\text {h }} 4^{\prime}$ nach 1 cen starke Uebelkeit nit Würgen.

Bis $2^{\text {h }} 45^{\prime} 11 \mathrm{ccm}$ Blutdruck $80 \mathrm{mn}$, Puls 164, Respiration 26 .

Bis $3^{\text {h }} 18^{\prime} 15 \mathrm{ccm}$ noch öfters starke Würgen, Puls kaun fühlbar, am Herzen 224.

$3^{\text {h }} 30^{\prime}$ Lösung $1: 1$ es laufen noch 4,5 cens ein; Ucbelkeit mit Würgen; friert stark. Puls kaum zu fühl $\bullet$ n.

Da weitercr $\mathrm{Zu}$ fluß stockt, Abbruch des Versuches. Giesanitadrenalinmenga $6 \mathrm{mg}$.

$7^{\text {ht }}$ abends Blut druck $70 \mathrm{~mm}$, Puls 132, ord.: Digitalis, Kampfer.

28. Juli Puls regelmäßig, gegen 100 , Blutdruck 135. Allmähliche Wiederherstellung der Konpensatiol.

10. September be schwerdefrei entlassen.

28. September wieder nitleichtenKoinpensationsstörungen aufgenommen, dic sich rasch beseitigen lassen.
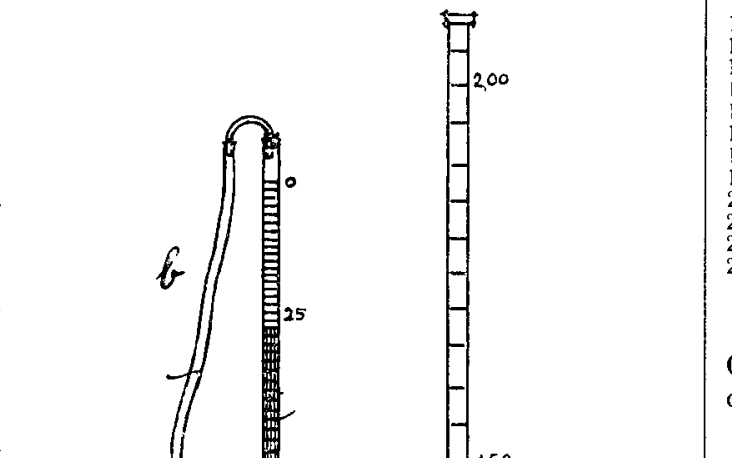

\begin{tabular}{|c|c|c|c|c|c|c|}
\hline & Zeit & $\begin{array}{l}\text { Ueber- } \\
\text { druck }\end{array}$ & $\begin{array}{l}\text { Stand } \\
\text { in Burelte }\end{array}$ & Puls & $\begin{array}{c}\text { systol. } \\
\text { Blutdruck }\end{array}$ & Atmung \\
\hline & ${ }_{15}^{13} \mathrm{Mins}$ & $65 \mathrm{c}$ & $\begin{array}{l}29,2 \\
29,8\end{array}$ & $\begin{array}{l}\text { nicht fühlbar } \\
160\end{array}$ & = & 4.) \\
\hline 1 1 & $\begin{array}{ll}19 & n \\
23 & n\end{array}$ & $\begin{array}{c}100 \mathrm{c} \\
n\end{array}$ & $\begin{array}{l}31,0 \\
33,6\end{array}$ & $-. .0-2$ & 105 & 40 \\
\hline & $\begin{array}{l}30 \\
39\end{array}$ & $n$ & $\begin{array}{l}37,2 \\
42,5\end{array}$ & $\begin{array}{l}160 \\
180\end{array}$ & 93 & $\begin{array}{l}40 \\
40\end{array}$ \\
\hline i & 42 & ; & 44,0 & Neufüllung & der Burette & \\
\hline & - & $"$ & $\begin{array}{r}3,0 \\
1,3,2\end{array}$ & $\begin{array}{l}163 \\
180\end{array}$ & $\begin{array}{r}95 \\
100\end{array}$ & $\begin{array}{l}40 \\
40\end{array}$ \\
\hline $\begin{array}{l}2 \\
2\end{array}$ & $\begin{array}{l}30 \\
45\end{array}$ & & $\begin{array}{l}34,0 \\
45\end{array}$ & $\begin{array}{l}164 \\
180\end{array}$ & $\begin{array}{l}80 \\
70\end{array}$ & $\begin{array}{l}40 \\
40\end{array}$ \\
\hline & $51 n$ & & 49,0 & & & \\
\hline
\end{tabular}

Concretio pericardii cum conde. Die Infektion des Bauchfells war durch das nit der gangränösen linken Lunge verwashsene Zwerchfell erfolgt.

Fall 4. 40jähriger Mann; 16. August wegen Typhus abdominalis im Anfange der dritten Woche aufgenommen. 17. August rostfarbener Auswurf; links hinten unten handbreite Dämpfung mit Bronchialatmen und Knistern, Tenıperatur 40,5, Puls 124, Abdomen nüßig aufgetrieben.

21. August. Leib stark meteoristisch aufgetrieben; keine Däınpfung der abhängigen Partien, keine Zeichen von Peritonitis. Pneumonie unverändert. Bei Abendvisite Temperatur 38,9, Puls 148, öfters aussetzend, aber Blutdruck bei einviertelstündlichen Kampferölinjektioneı $135 \mathrm{num} \mathrm{Hg}$; $9 \mathrm{Uhr}$ abends Puls 180, Atmung 52, Blutdruck $10 \mathrm{~mm} \mathrm{Hg}$; deshalb sofort Suprarenininfusion in eine Hautvene an rechten inneren Knöchel; Suprarenin 1:3 NaCl-lösung:

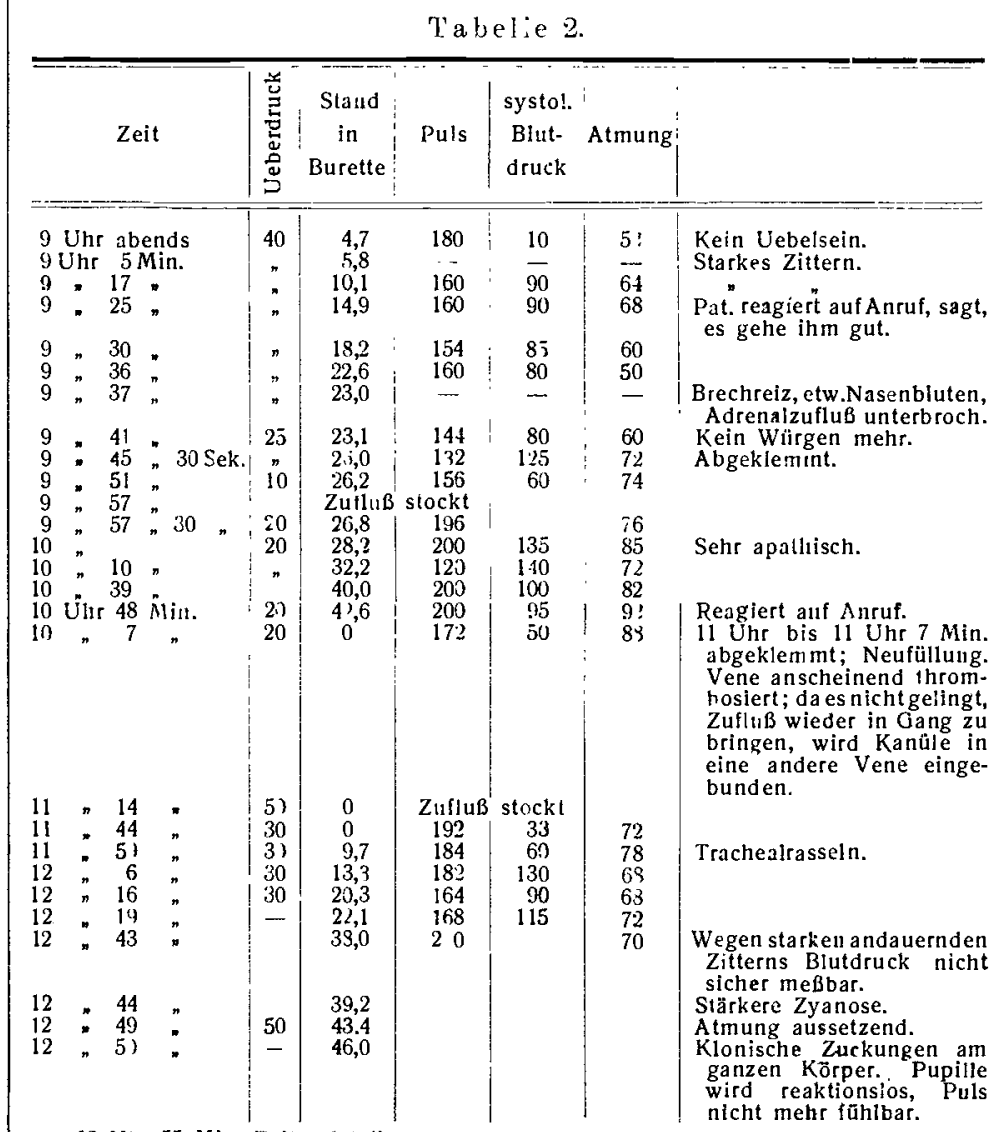

12 Uhr 55 Min. Exitus letalis.

Im ganzen sind in 3 Stunden 55 Minuten eingeflossen $87,8 \mathrm{ccm}$ Suprarenin-Kochsalzlösung, entsprechend 0,0219 g Suprareninum syntheticum.

Die Sektion (Dr. Binder) ergab Ileotyphus, hypostatische Pneumonie des linken Unterlappens, Herzmuskulatur schwach entwickelt, in den Papillarmuskeln streifenförmige Blutungen, in den Spitzen der Papillarmuskeln schwielige Einlagerungen; eitrige Bronchitis.

Fall 5. 49 jähriger, sehr dekrepider Tabiker wird wegen jahrelang dauernder, heftigster gastrischer Krisen der Försterschen Operation unterworfen (einzeitig), am 23. August 1910. Eine halbe Stunde nach Beginn der Narkose Asphyxie, sodaß künstliche Atmung nötig wird. Puls kaum mehr zu fühlen.

Auf Wunsch des Operateurs (Geh.-Rat Heusner) deshalb intravenöse Suprarenininfusion in eine Hautvene am rechten äußeren Malleolus. Suprarenin 1:3 $\mathrm{NaCl}$-lösung. 
Tabelle 3

\begin{tabular}{|c|c|c|c|c|c|c|}
\hline Zeit & $\begin{array}{l}\text { Ueber- } \\
\text { druck }\end{array}$ & $\begin{array}{c}\text { Stand } \\
\text { in } \\
\text { Burette }\end{array}$ & Puls & $\begin{array}{c}\text { sysiol. } \\
\text { Blut- } \\
\text { druck } \\
\text { in } \mathrm{mm} \\
\mathrm{Hg} .\end{array}$ & Atmuns & \\
\hline 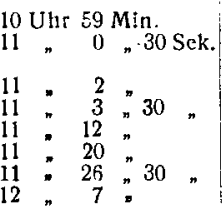 & $\begin{array}{l}25 \\
= \\
= \\
=\end{array}$ & $\begin{array}{r}8,0 \\
8,6 \\
9,8 \\
12,2 \\
14,6 \\
30,8\end{array}$ & $\begin{array}{l}160 \\
132 \\
190 \\
190 \\
184 \\
188\end{array}$ & $\begin{array}{l}135 \\
115\end{array}$ & $\begin{array}{l}36 \\
36 \\
40 \\
40 \\
40 \\
48\end{array}$ & $\begin{array}{l}\text { Puls kaum fühlbar. } \\
\text { Wurnde beginnt stark z } \\
\text { bluten, kein Uebelsein } \\
\text { Plils ist gut fühlbar. } \\
\text { Eröffnung der Dura. } \\
\text { Puls ist kräftig. } \\
\text { Operation beendet. }\end{array}$ \\
\hline
\end{tabular}

Im ganzen sind 25,8 Suprarenin-Kochsalzmischung, entsprechend $0,00645 \mathrm{~g}$ Suprareninum syntheticum, eingeflossen. Zunächst ungestörter Verlauf. Am 31. August Exitus an hämorrhagisch-diphtherischer Cystitis und Lingengangrän.

Dies sind meine wenigen bisherigen Versuche; ich hoffe, bald weitere Mitteilungen bringen zu können. Zu Schlüssen für oder gegen die Dauerinfusionen berechtigen dieselben naturgemäß nicht; dazu bedarf es weiterer zahlreicher Beobachtungen an sorgfältig ausgesuchten Fällen.

Technisch ist die Methode nach meiner Ueberzeugung auch bei langer Dauer der Anwendung in jedem Krankenhause leicht durchführbar; der Patient bedarf allerdings, besonders bei Störungen des Sensoriums, dauernder Veberwachung durch zuverlässiges Pflegepersonal: öftere Kontrolle des Blutdruckes durch den Arzt erscheint mir wünschenswert. Ich glaube und hoffe, daß dic Methode sich Bürgerrecht in unseren klinischen therapeutischen Manipulationen erwerben wird.

Die nächste Aufgabe wird es sein, das Indikationsgebiet für die Dauerinfusion genau zu umgrenzen. Am aussichtsreichsten scheint mir nach den bisherigen günstigen Mitteilungen in der Literatur über intravenöse Adrenalinanwendung die Verwendung der Dauerinfusion auf chirurgischem Gebiete zu sein (schwere Herzstörungen während der Narkose, schwere langdauernde postoperative Kollapse, besonders nach Laparotomien, ev. auch zur Bekämpfung der peritonitischen Blutdrucksenkung etc.).

Von inneren Krankheiten dürften $\mathrm{m}$. E. in erster Linie schwere Kollapse bei akuten Infektionskrankheiten, bei denen die Vasomotorenlähmung eine so bedeutungsvolle Rolle spielt, in den Bereich der therapeutischen Versuche mit Adrenalindauerinfusion zu ziehen sein, also vor allem bei Scharlach, Diphtherie, Typhus, ev. auch bei Pneumonie. Aber auch bei schweren, besonders akuten Kompensationsstörungen im Verlaufe von chronischen Herzerkrankungen dürfte trotz des starken Ueberwiegens der Vasomotorenwirkung des Adrenalins über die eigentliche Herzwirkung nach den bisherigen günstigen Literaturberichten über Adrenalinanwendung von Dauerinfusion vielleicht mit Vorteil Gebrauch zu machen sein. Dabei dürfte allerdings auf Gegenanzeigen zu achten sein: A priori ist es denkbar, daß bei stark geschwächtem Herzen eine brüske Erhöhung der peripherischen Widerstände vielleicht ein schnelleres Erlahmen des Herzens zur Folge haben kann. Ev. dürfte sich für Fälle, welche Befürchtungen in dieser Richtung aufkommen lassen, die Kombination mit einem kräftig und schnell wirkenden Cardiotonicum (Strophanthin, Digalen, Kampfer) empfehlen.

Wie unsere beiden ersten Fälle zeigen, machen sich zuweilen als üble Nebenwirkungen Uebelkeit und Erbrechen geltend, vielleicht ein Hinweis auf die Vaguswirkung des Adrenalins; auch in einem dritten Falle kam es vorïbergehend zu Brechreiz.

Besonders aber dürfte in Zukunft darauf $\mathrm{zu}$ achten sein, ob sich bei günstig verlaufenden Fällen im weiteren Decursus Hinweise auf stattgehabte organische Schädigung der Gefäßwände geltend machen, wie man nach Literaturangaben befürchten könnte. Unsere Fälle 2 und 5 scheinen diese Besorgnis nicht $\mathrm{zu}$ rechtfertigen.

Zusammenfassung. Im Tierversuch bringt kontinuierliche langdauernde intravenöse Adrenalininfusion gleichmäBige Blutdrucksteigerung während der ganzen Infucionsdauer hervor; ein Nachlassen der Wirkung tritt nicht ein.

Die Uebertragung der Methode ans Krankenbett (im klinischen Betriebe) wird angeregt. Der Anwendungsbereich derselben, Gegenanzeigen, zweckentsprechende Dosierung etc. werden sich erst auf Grund breiterer Erfahrung präzisieren lassen 